XLIII.

\title{
REPORT OF A CASE OF ACUTE MASTOIDITIS WITH \\ INFLUENZAL MENINGITIS. TREATMENT BY \\ OPERATION ON THE MASTOID AND ANTI- \\ INFLUENZAL SERUM, FOLLOWED BY \\ BRAIN ABSCESS - OPERATION - \\ RECOVERY.
}

By Francis R. Packard, M. D.,

Philadelphia.

E. C., eleven years old, was taken ill on Wednesday, October 2,1913 , on which day she came home from school, having been exposed to a severe wetting in a rain storm. That night she had a chill, followed by fever. A few days later she developed a pain in her right ear, which was followed by vomiting and headache. I was called in to see her, in consultation, by Dr. Knipe, the family physician, on the 12th of October, ten days after her initial chill. She complained of severe headache. Her temperature was $102^{\circ}$, pulse 120 , and she seemed very irritable and had some photophobia. I incised her right ear drum and got a great quantity of pus from the right middle ear.

The next day, October 13th, she was admitted to the Pennsylvania Hospital, and I opened her right mastoid. Before the operation she was seen by Dr. Arthur Newlin, who agreed with me that she was suffering from meningitis, with acute mastoiditis. The child lay in bed, on the left side, with head retracted, crying out sharply from time to time. The pupils were rather large, reacted to light, and were equal. Her heart and lungs were negative. Kernig's sign was present to a slight degree. Her blood count showed 26,000 leucocytes, ninety per cent of which were polymorphonuclears.

Upon opening the right mastoid, which I did under ether, the process was found to be extremely cellular, and the bone very soft in consistence, a small amount of pus in the antrum 
and in the cells towards the tip. Whilst she was under ether a lumbar puncture was made in the fourth interspace by $\mathrm{Dr}$. Billings, and twenty cubic centimeters of spinal fluid, under great pressure, was removed. The patient reacted quietly after the operation. Owing to the appearance of the spinal fluid and the history of the case, it was decided to give the patient the antiinfluenzal serum of Dr. Flexner, of the Rockefeller Institute, of which Dr. Richardson had some in the Ayer Clinical Laboratory. This was done the same evening, and fifteen cubic centimeters of the spinal fluid were withdrawn and thirty cubic centimeters of the antiinfluenzal serum were injected under ethylchlorid anesthesia.

On October 14th the following report was received from Dr. Richardson of the Ayer Clinical Laboratory of the Pennsylvania Hospital:

"The spinal fluid presented the typical picture of that from a case of acute meningitis, being very turbid. Noguchi's butyric acid test was positive, and Fehling's copper solution was not reduced. Microscopic examination showed many pus cells, almost all of which were polynuclears. There were present some organisms which resembled B. influenzx. Some of the sediment was cultured on human blood agar. After twentyfour hours there appeared several fine white colonies, made up of a small gram negative bacillus. These on further study proved to be $B$. influenza."

The same day, October 14th, another lumbar puncture was made by Dr. Billings under ethylchlorid anesthesia, and thirty cubic centimeters of spinal fluid were withdrawn and thirty cubic centimeters of the antiinfluenzal serum injected.

On October 15th the patient was very much more comfortable, still had some photophobia, and Kernig's sign was marked in the right leg, with much less rigidity of the neck, and the child was brighter. She was again given ethylchlorid and about thirty cubic centimeters of spinal fluid were withdrawn by lumbar puncture from the spinal cord, followed by the injection of thirty cubic centimeters of the antiinfluenzal serum.

On the 16th the child's condition was much the same, except that she was much more irritable. The lumbar puncture and the injection of the antiinfluenzal serum was repeated, but 
very little fluid was withdrawn from the spinal column, and it was practically clear. Thirty cubic centimeters of the antiinfluenzal serum were injected.

On October 17th the child's condition showed most distinct improvement. She was cheerful and bright, with no rigidity of the neck, and Kernig's sign had practically disappeared. There was still some twitching of the muscles in certain parts of the body during sleep. This was the date of the last injection that the child received.

Dr. Richardson furnished me with the accompanying laboratory note as to his findings throughout the period in which the child received injections of the antiinfluenzal serum.

"The further examination of fluid during treatment with the serum showed a gradually improving condition. There were no organisms found after the first specimen. The pus cells decreased in number, and the butyric acid and Fehling's tests later became negative. Several specimens of spinal fluid were examiner. The last showed an almost normal fluid with only slightly increased number of white cells. The butyric acid test showed only the smallest possible flocculent precipitate, while Fehling's solution was reduced."

Up to this date the pus from the ear discharge showed a pure culture of staphylococcus aureus.

From this date, October 17 th, which was four days subsequent to the operation and to the first injection of the antiinfluenzal serum, the child's case followed an uneventful course, until October 29, 1913. Her mastoid wound had practically healed, there being only a small surface on the lower portion which had not closed over. There was a slight amount of serous discharge from the external auditory canal.

On October 29th the child developed a slight temperature $\left(100^{\circ}\right)$, accompanied by some rapidity of the pulse. She complained of headache, and seemed very irritable. She had no Kernig's sign and no rigidity of the neck. Two days later, November 1st, she had several vomiting spells of a projectile character, and also seemed somewhat drowsy. Dr. William $T$. Shoemaker examined the eye grounds, and reported that the right optic disc was very hazy. Dr. Robert G. Le Conte saw the child in consultation with Dr. Arthur Newlin and my. self. It was decided that the symptoms were indicative of 
brain abscess, and on November 3, 1913, Dr. Le Conte and myself opened the cranium. On exposing the dura it was found to be very tense and bulging, and a large abscess cavity was found in the substance of the temporosphenoidal lobe. - In the course of the operation the lateral sinus was accidentally punctured, but the hemorrhage was controlled without trouble. A rubber drainage tube was inserted into the wound, which was packed with plain gauze.

The subsequent course of the case affords nothing of any particular interest. Drainage of the wound was done away with on November 11 th, eight days subsequent to the operation, and the patient was discharged, cured, from the hospital on December 7, 1913.

Dr. Richardson's report on the pus from the brain abscess showed a pure culture of streptococcus.

I have reported this case because it seems to me that the primary meningitis was undoubtedly influenzal and that the antiinfluenzal serum proved efficacious in its relief. The results following its administration were so striking that they seemed to be conclusive. I think it is also interesting to note that the laboratory report showed that the pus from the ear discharge was a pure culture of staphylococcus; the culture from the spinal cord showed the influenzal bacillus; and the pus from the brain abscess showed a pure culture of streptococcus. 